\title{
KOMISCHES UNGLÜCK: \\ ERZÄHLER UND ERZÄHLFIGUREN SAMUEL BECKETTS
}

\section{Peter Brockmeier}

Die Anregung für das Thema unseres Kolloquiums finden wir in einer Szene von Fin de partie. Hamm fühlt sich durch das Gerede seiner Eltern, Nagg und Nell, gestört; er möchte schlafen und, wenn er schliefe, träumen oder in seinem Sinn leben: "Si je dormais je ferais peut-être l'amour. J'irais dans les bois. Je verrais... le ciel, la terre. Je courrais. On me poursuivrait. Je m'enfuirais (Un temps.) Nature! (Un temps.) Il y a une goutte d'eau dans ma tête. (Un temps.) Un coeur, un coeur dans ma tête."1

Verweilen wir einen Moment bei der Spannung dieser Aussage: Selbsthingabe, Selbstaufgabe, Flucht vor dem "Herzeleid und den tausend naturgegebenen Erschütterungen"2; Sehnsucht nach der Aufnahme oder Auflösung des Ich in einer vielleicht herrlich leuchtenden, Liebesglück stiftenden "Natur"3 - diese Traumvision oder Traumerinnerung ist die Sehnsucht eines anderen, eines zweiten Ich. Nagg findet die Vorstellung eines Ich komisch, das sich teilt oder im eigenen Kopf mit sich selbst spricht: "Il glousse précautionneusement." ${ }^{4}$ Nell glaubt, nicht darüber lachen zu dürfen; sie gibt aber zu: "Rien n'est plus drôle que le malheur". ${ }^{5}$ Nur weil wir uns an das Unglück wie an einen wiederholt erzählten Witz gewöhnen, finden wir es immer wieder gut, lachen aber nicht mehr darüber. ${ }^{6}$ Beckett hat 1967 , bei den Proben im Berliner Schiller-Theater, die Aussage Nells: "Nichts ist komischer

1 S. Beckett, Fin de partie suivi de Actes sans paroles, Paris 1957, S. 30.

2 W. Shakespeare, Hamlet, zweispr. Ausgabe v. H.M. Klein, Stuttgart 1984; 3.1, v. 60ff.

3 Vgl. etwa J.W. v. Goethe, Mailied, in: ders., Sämtliche Werke, hg. v. E. Beutler, München 1977; Bd. 1, S. 52 f.

4 Beckett, Fin de partie, S. 33.

5 Ebd.

6 Ebd., S. 33f. 
als das Unglück" als den für ihn wichtigsten Satz des Stücks bezeichnet. ${ }^{7}$ Die komische Wirkung der Texte Becketts - so lehrt uns Hamm - liegt darin begründet, daß Erzähler oder Erzählfiguren als Sprecher und auktoriale Instanzen die Texte eröffnen, daß sie sich selbstreflektierend verdoppeln oder vervielfältigen und daß sie als ein und dasselbe Erzähler-Ich den Text verlassen oder ihn beschließen.

Der Erzähler Becketts ist wie jeder Erzähler eines literarischen Textes allmächtig, weil er phantasierend spricht; er weiß aber, daß er nur von sich und über sich selbst spricht und daß diese Fragen an sich selbst nicht zu beantworten sind.

Ich möchte zuerst Becketts Konzept des Erzählers und seine Theorie des literarischen Schreibens - "l'autologie créatrice" - vorstellen. Anschließend werde ich mit Hilfe der Theorie der Kreativität von Arthur Koestler zu begründen versuchen, daß der schöpferische Eigensinn unlösbar mit dem "humour" verbunden ist. Inwieweit der in der Tat tiefgründige Witz des Solipsismus Becketts Erzählfiguren und Erzähler, also seine Schreibweise, innovativ bestimmt, möchte ich zuletzt noch anhand eines Textes aus dem Roman Murphy erläutern.

1.

Ein Muster für den allwissenden und allmächtigen Erzähler hat Balzac geschaffen. Weit entfernt, den Erzähler der Comédie humaine auf die Rolle eines "Registrators von Gut und Böse"8 zu reduzieren, überträgt Balzac ihm vielmehr die Aufgabe, Sinn und Bedeutung der gesellschaftsgeschichtlichen Erscheinungen und ihre Ursachen zu erfassen: "surprendre le sens caché dans cet immense assemblage de figures, de passions et d'événements". 9 Der

7 M. Haerdter, "Samuel Beckett inszeniert das Endspiel"; in: Materialien zu Becketts 'Endspiel', Frankfurt a.M., ${ }^{21970, ~ S . ~} 50$.

8 H. de Balzac, La Comédie humaine, hg. v. M. Bouteron, Paris 1951, Bd. 1, "Avant-propos", S. 7.

9 Ebd. 
Erzähler soll, im Unterschied zum antiquarisch sammelnden Historiker, die Naturgesetze der Entwicklung, "les principes naturels", sichtbar machen; er soll die Antriebskraft der Gesellschaft, "ce moteur social", staatsphilosophisch durchdringen, um erkennbar werden $\mathrm{zu}$ lassen, wie weit sich die Gesellschaftsformationen von den "ewigen Wahrheiten", nämlich Religion und Monarchie, entfernen oder sich ihnen annähern. ${ }^{10}$ Der Erzähler Balzacs, Konstrukteur einer in sich geschlossenen Welt, erschien Beckett schon in einem Manuskript aus dem Jahr 1932 als ein Zauberkünstler, der so tut, als könnte er mit scheinbar dauerhaften Werten jonglieren, sie miteinander verschmelzen, vergrößern oder verkleinern; sein Material kalkuliert er aufs genaueste, seine Charaktere sind mechanische Dummköpfe. ${ }^{11}$ Nachdem Beckett seine Roman-Trilogie veröffentlicht hatte, gab er Israel Shenker zu verstehen, daß er seine Erzähler aus der "Erfahrung eines Nicht-Wissenden, eines Nicht-Könnenden" sprechen ließ. Im Unterschied zu Joyce, "einem hervorragenden Manipulator seines Materials", der als Künstler "Allwissenheit und Allmacht" erstrebte, schrieb Beckett ohne die Vorgabe, Herr seines Materials zu sein. Er soll zu Shenker gesagt haben: "I'm working with impotence, ignorance." ${ }^{12}$ Er meinte damit doch wohl: Ich als Autor setze einen Erzähler ein, der spricht oder formuliert, als wäre er ohnmächtig und

10 Ebd., S. 7, 9.

11 S. Beckett, Dream of Fair to Middling Women, hg. v. E. O'Brien u. E. Fournier, Dublin 1992, S. 119f.: "So all novelist has to do is to bind his material in a spell, item after item, and juggle politely with irrefragable values, values that can assimilate other values like in kind and be assimilated by them, that can increase and decrease in virtue of an unreal permanence of quality. To read Balzac is to receive the impression of a chloroformed world. He is absolute master of his material, he can do what he likes with it, he can foresee and calculate its least vicissitude, he can write the end of his book before he has finished the first paragraph, because he has turned all his creatures into clockwork cabbages and can rely on their staying put wherever needed or staying going at whatever speed in whatever direction he chooses. [...] We all love and lick up Balzac, we lap it up and say it is wonderful, but why call a distillation of Euclid and Perrault Scenes from Life? Why buman comedy?"

12 I. Shenker, "Moody Man of Letters", in: New York Times, 6.5.1956, 2 X, S. 3. 
unwissend. Den Autor Beckett wird man, ohne allzu heftigen Widerspruch befürchten zu müssen, als "poeta doctissimus" bezeichnen dürfen.

In einem posthum veröffentlichten Typoskript des Jahres 1938, das den Titel "Les Deux Besoins" trägt ${ }^{13}$, können wir nachlesen, daß der Erzähler Becketts aus der Perspektive seines beschränkten subjektiven Wissens weder Wahrheiten sucht noch dauerhafte Werte manipuliert, sondern daß er seine "geistigen Vorgänge" und ihren "spezifischen Erlebnisgehalt"14 wiederzugeben versucht. Er formuliert, was er will, denkt, tut, leidet, ist. Wenn Beckett ${ }^{15}$ in diesem Zusammenhang von "dem Künstler" spricht, der den wenigen, für die er existiert, einen Blick auf die "monotone centralité" dessen eröffnet, was "ein jeder" will, denkt, leidet, so versteht er unter dem Künstler natürlich auch den Erzähler im Text: Das Ich des Erzählers setzt ausdrücklich oder unausdrücklich alle Pronomen oder Eigennamen als Varianten seiner selbst. ${ }^{16}$ Wir sollten Belacqua, Murphy, Watt, Sam oder Sapo, Macmann, Lemuel, Worm, Mahood oder Pim, Bem, Bom, Kram und alle übrigen Namen in Becketts Texten als Setzungen des Erzählers, als Vervielfältigungen der Erzählerinstanz begreifen, die ohne jenen nicht wären, sowenig wie der Erzähler ohne den Autor.

Die Lektüre Becketts sollten wir uns nicht dadurch erschweren, daß wir übertrieben konsequent und unnachgiebig den Ich-Erzähler vom NullErzähler - oder dem auktorialen Erzähler - und den Erzählfiguren unterscheiden; wir könnten den Solipsismus übersehen. Denn Beckett hat den Schaffensdrang als ein "Zusammenziehen des Geistes, ein Hinuntersteigen"17,

13 In: S. Beckett, Disjecta. Miscellaneous Writings and a Dramatic Fragment, hg. v. R. Cohn, London 1983, S. 55-57.

14 Th. Metzinger, Subjekt und Selbstmodell. Die Perspektivität phänomenalen Bewußtseins vor dem Hintergrund einer naturalistischen Theorie mentaler Repräsentation, Paderborn 1993, S. 20.

15 Beckett, "Les Deux Besoins", a.a.O., S. 55.

16 Vgl. K. Weimar, Enayklopädie der Literaturwissenschaft, München 1980, \$S 245, 232, S. 135 , 129.

17 S. Beckett, Proust and Three Dialogues with Georges Dubuit, London 1976, S. 65f. Dt. Übertragung des Aufsatzes über Proust von M. u. P. Pörtner, Zürich 1960, S. 45. 
als eine Auseinandersetzung des Ichs mit sich selbst, als 'schöpferisches Selbstgespräch' - "autologie créatrice" - bezeichnet. Nach der Abfassung des Aufsatzes über Proust (1931) veranschaulichte er diese kreative Selbstbetrachtung oder Innenschau mit einem Diagramm, das aus zwei gleichschenkligen Dreiecken besteht.

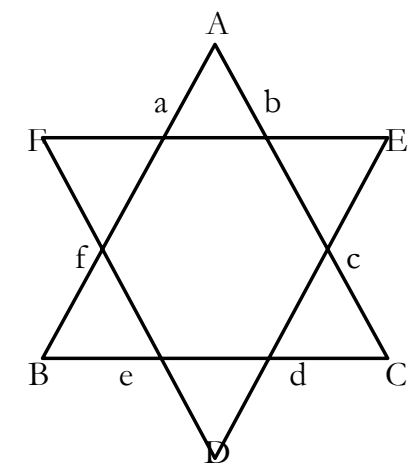

Besoin d'avoir besoin (DEF) et besoin dont on a besoin (ABC), conscience du besoin d'avoir besoin (ab) et conscience du besoin dont on a besoin - dont on avait besoin (de), issue du chaos de vouloir voir (Aab) et entrée dans le néant d'avoir vu (Dde), déclenchement et fin de l'autologie créatrice (abcdef). Voilà par exemple une façon comme une autre d'indiquer les limites entre lesquelles l'artiste se met à la question, se met en question, se résout en questions, en questions rhétoriques sans fonction oratoire. ${ }^{18}$

Der Künstler, der in Texten als Erzähler auftritt, bringt das zum Ausdruck, was er undeutlich in seinem eigenen Bewußtsein hat wahrnehmen können. Er beschäftigt sich mit Fragen, die er an sich selbst stellt, denen er sich selbst unterzieht, mit denen er sich selbst in Frage stellt; er löst sich in "rhetorischen Fragen" auf, die keine "rednerische Funktion" erfüllen. Unter dieser "rednerischen Funktion" verstehe ich Anklage und Verteidigung, das "genus iudiciale", Zuraten oder Abraten, das "genus deliberativum", Lob und Tadel, das "genus demonstrativum". Wir sollten allerdings beachten, daß Beckett nicht die Mitteilungsfunktion von Texten aufgegeben hat; er hat mit der rednerischen in erster Linie auf die konative Funktion des

18 Beckett, "Les Deux Besoins", a.a.O., S. 56. 
Kommunikationsmodells verzichtet. Das Modell, das er in dem Aufsatz "Les Deux Besoins" entworfen hat, muß man sich nach seinen Worten als Zwölfflächner vorstellen. Es ist also das Modell einer "boîte crânienne". ${ }^{19}$ Die Selbstbezogenheit des künstlerischen Schaffens oder des Erzählens, das Werk oder der Text als "Folge reiner Fragen" oder "cri à blanc", haben nichts mit der Wissenschaft, der Theologie oder den eindeutigen Entscheidungen zwischen Ja und Nein zu tun. ${ }^{20}$ Der Künstler schafft im Rahmen seines reflektierenden Bewußtseins: "Excluant et exclu, il traverse l'élément social, sans le juger."21 Beckett hat sein Modell des schöpferischen Selbstdenkens oder Selbstgesprächs dank der Philosophie von Arnold Geulincx (1624-1669), eines flämischen Cartesianers, entwickelt. ${ }^{22}$

Die Quelle des Komischen in Becketts Texten ist in der konsequenten Eigensinnigkeit des Selbst-Denkens oder Selbst-Erzählens zu suchen: Ausschließend und ausgeschlossen bewegt der Erzähler sich durch traditionelle sinnstiftende Diskurse und Schreibweisen. Um die komische Wirkung dieses literarischen Verfahrens zu begründen, greife ich auf Arthur Koestlers Theorie des Komischen zurück, die ein zentraler Bestandteil seiner Theorie der Kreativität ist. ${ }^{23}$ Ich möchte auf diese Weise die Frage aufgreifen, die Dieter Lamping gestellt hat: "Ist Komik harmlos?" Becketts Texte bieten ein

19 Beckett, Le Monde et le pantalon [1945/46], Paris, 1989, S. 28.

20 Beckett, "Les Deux Besoins", a.a.O., S. 56f.

21 Beckett, "Le Concentrisme" [etwa 1930], in: Disjecta, S. 38f.

22 Vgl. D. Bair, Samuel Beckett. Eine Biographie, dt. v. W. Peterich, Reinbek bei Hamburg 1994, S. 135.

23 A. Koestler, The Act of Creation, Sydney, Toronto, New York 1964. Dt. Ausgabe: Der göttliche Funke, Bern, München 1966. Ich stütze mich im folgenden auf die leicht zugängliche Zusammenfassung der Theorie in: A. Koestler, Der Mensch - Irrläufer der Evolution. Die Kluft zwischen Denken und Handeln. Eine Anatomie menschlicher Vernunft und Unvernunft, aus d. Engl. v. J. Abel, Frankfurt a.M. 1989. 
anschauliches Beispiel dafür, wie das Komische gewiß nicht erst in der Literatur des 20. Jahrhunderts seine Unschuld verlieren kann. ${ }^{24}$

Koestler führte alle schöpferischen Tätigkeiten - das Kunstschaffen, das wissenschaftliche Forschen, den Witz - auf ein "gemeinsames Grundschema" zurück. ${ }^{25}$ Witz oder Humor dienen hierbei als der "Hintereingang zur Domäne der Kreativität"; der Witz ist ein einzigartiges Beispiel, insofern ein komplexer intellektueller Reiz eine "einfache körperliche Reaktion auslöst - den Lachreflex". 26 Die "Grammatik", nach der die witzige Formulierung, die wissenschaftliche Entdeckung und die Produktion künstlerischer Illusionen Karikatur, Diagramm, Portrait - verfahren, nannte Koestler "Bisoziation": "das Wahrnehmen einer Situation oder eines Ereignisses in zwei sich ausschließenden Assoziationsrahmen."27 Der Komiker formuliert ein Paradoxon; der Wissenschaftler entdeckt die Lösung eines Paradoxons; kraft seiner Phantasie spielt der Künstler eine solche Lösung vor. Ein uns allen geläufiges und wohl noch nicht gelöstes Paradoxon, also ein "bisoziatives Muster", der "Zusammenstoß unvereinbarer Verhaltenskodizes", ist die Dichotomie von Mensch und Tier. Diese kann auf komische, unheimlich ernste oder wissenschaftliche Weise untersucht werden: Donald Duck, Kafkas Die Verwandlung, die Rattenversuche der Psychologen. ${ }^{28}$ Die Auswirkungen der Paarung von Mensch und Tier sind austauschbar. Denken wir an Estragons und Vladimirs vergnügliche und empörte Reaktion gegenüber dem Paar Pozzo

24 D. Lamping, "Ist Komik harmlos? Zu einer Theorie der literarischen Komik und der komischen Literatur", in: Literatur für Leser, 94/2, S. 64f.: "Das Komische in der modernen Literatur ist aber durch seine Ambivalenzen nicht bloß komplexer, sondern auch problematischer geworden, gerade in moralischer Hinsicht. Die Begrenzung auf das harmlos Humoristische war zugleich eine auf das moralisch Unverfängliche, das ethisch Erlaubte. Durch die Emanzipation von ihm hat sich die Komik nun z.B. auch auf die Aggression, das Ressentiment, ja den Schrecken und die Gewalt einlassen können. Dadurch mag sie unmoralischer geworden sein. [...] Mit der Harmlosigkeit hat das Komische seine Fesseln - und seine Unschuld verloren."

25 Koestler, Der Mensch - Irrläufer der Evolution, S. 131.

26 Ebd., S. 155.

27 Ebd.

28 Ebd., S. 154. 
und Lucky, der als Lasttier und Diener vorgeführt wird: "C'est une honte." "Charmante soirée."29

Wenn Koestler die "Kreativität in der Wissenschaft" als "Kunst" bezeichnete, "zwei und zwei so zusammenzuzählen, daß dabei fünf herauskommt"30, so fällt es nicht allzu schwer, den Übergang und den Zusammenhang zwischen der bisoziierenden Wahrnehmungsfähigkeit eines Erzählers und dem Staunen eines Wissenschaftlers nachzuvollziehen, die sich gemeinsam mit dem Leser oder dem Besucher im Laboratorium über ein Experiment an Lebewesen beugen, über "Eine Bleibe, wo Körper immerzu suchen, jeder seinen Verwaiser." 31

Der Erzähler von Le Dépeupleur (1970) ist ein "denkendes Wesen", das kalt alle "Gegebenheiten und Offensichtlichkeiten" des mit dem Text angelegten Experimentes wiedergibt. Am Ende seiner Untersuchung kommt er, wie es bei wissenschaftlichen Untersuchungen selbstverständlich ist, zu einem vorläufigen Ergebnis: Man sollte die regungslosen Körper im Zylinder besser mit dem sachlichen Terminus "Blinde" als mit der "unangenehm pathetischen" oder: literarischen Metapher "Besiegte" bezeichnen. ${ }^{32}$ Die Bisoziation der nüchternen Vorstellung mit einer zweiten, die Mitgefühl oder gar Ehrfurcht erheischen könnte, ist als eine Kollision der Beobachtung des Erzählers mit seinen Geschöpfen zu bewerten: Das hingebungsvolle ernste "Ah" der Einfühlung wird mit einem nüchtern distanzierenden "Aha" oder gar ironischen "Haha" kontrastiert. Die metasprachliche Reflexion, die ich soeben zitiert habe, ist symptomatisch für das Bestreben der Erzähler Becketts, die "ästhetische Verzückung"33 aufzuheben. Koestler ${ }^{34}$ hat der Kunst die Funktion

29 S. Beckett, En attendant Godot, Paris 1952, S. 43, 56.

30 Koestler, Der Mensch - Irrläufer der Evolution, S. 157.

31 S. Beckett, Le Dépeupleur/ Der Verwaiser. Französisch und deutsch. Deutsche Übertragung Elmar Tophoven, Frankfurt a.M. 1972, S. 6-7.

32 Ebd., S. 78-9.

33 Koestler, Der Mensch - Irrläufer der Evolution, S. 166; vgl. die schematische Darstellung der drei Bereiche der Kreativität auf S. 132.

34 Ebd., S. $166 f$. 
zugeschrieben, das Bewußtsein zu erweitern und die engen Grenzen des Ich zu transzendieren; durch die Schaffung einer Illusion werden die teilnehmenden Emotionen der Leser, Zuschauer, Betrachter entfaltet und die selbstbehauptenden Bestrebungen gehemmt oder neutralisiert. ${ }^{35}$

Beckett entzieht seinem Text in der Regel die Stimuli, die eine kathartische Funktion ausüben oder die Entfaltung der "participatory emotions"36 im Leser bewirken können. Ich erinnere an den ersten Satz des Romans Murphy: "Die Sonne schien, da sie keine andere Wahl hatte, auf nichts Neues. Murphy saß, als ob es ihm frei stünde, im Schatten, in einer Gasse West Bromptons."37

Einer der letzten Texte, die Beckett in französischer Sprache publiziert hat, setzt wie folgt ein: "Tel quelqu'un ayant toute sa tête à nouveau dehors enfin ne sachant comment il ne s'y était trouvé que depuis peu avant de se demander s'il avait toute sa tête. Car de quelqu'un n'ayant pas toute sa tête peut-on raisonnablement affirmer qu'il se le demande et qui plus est sous peine d'incohérence s'acharne sur ce casse-tête avec tout ce qui lui reste de raison? Ce fut donc sous les espèces d'un être plus ou moins raisonnable qu'il émergea enfin ne sachant comment dans le monde extérieur et n'y avait à se demander s'il avait toute sa tête."38 Mit der Diversifikation, der Vervielfältigung, der rücksichtslosen Beseitigung oder Mißhandlung der Erzähler und Erzählfiguren verfolgte Beckett dasselbe Ziel: die Einfühlung des Lesers oder Zuschauers zu verhindern. Er benutzt die selbstbehauptende Tendenz, die Begründung von Witz und Humor, als generative Kapazität, wie sie einem jeden Text zugrunde liegt: "Wo immer [...] ein Pronomen (oder ein Eigenname oder ein Nomen, die durch ein Pronomen ersetzt werden können) im Text steht, da ist der Erzähler unausdrücklich mit dabei." 39 Als Text-Instanz vermag der von Natur sich

35 Koestler, Act of Creation, S. 306f.

36 Ebd., S. 307.

37 S. Beckett, Murphy, dt. von Elmar Tophoven, Hamburg 1994, S. 9.

38 S. Beckett, Soubresauts, Paris 1989, S. 17 f.

39 Weimar, Enzyklopädie der Literaturwissenschaft, \245, S. 135. 
selbst behauptende Erzähler hervorzubringen, was immer ihm beliebt: Karikatur, Diagramm, Metapher oder alles in einem - sofern er eben genial ist.

Der "Zusammenstoß unvereinbarer Verhaltenskodizes"40 wird also als Stimulus für komische oder tragische Texte und für wissenschaftliche Entdeckungen verwendet. Was den Begriff 'Humor' anbelangt, so sollten wir Koestler zufolge beachten, daß das "Spektrum des Humors" von groben bis zu subtilen Formen, vom albernen Schabernack über die Ironie bis zur Satire und zum schwarzen Humor reicht. Aber die Vielfalt der Stimuli oder der evozierten "widerstreitenden Gefühle" muß "eine grundlegende, unerläßliche Zutat enthalten: einen Impuls der Aggression oder Furcht, und sei er noch so schwach" - "eine momentane Anästhesie des Herzens". 41 Diesen Impuls bezeichnete Koestler als "self-asserting tendency". 42

Diese manifestiert sich in Becketts Texten als Selbstsetzung des Erzählers, dessen schöpferischer "Eigensinn" als ausdrückliche Absage an den genialischen "Großsinn" des allwissenden Erzählers zu verstehen ist. ${ }^{43}$ Die Selbstbehauptung bewirkt die kontinuierlich variierte Kollision des Erzählers mit sich selbst und auf diese Weise eine nicht immer offenkundige, aber generell mögliche Komik. Erinnern wir uns an die ersten Abschnitte des Romans More Pricks than Kicks: Belacqua in der Gegenwart von Beatrice und Dante steckt bis zum Hals in der Lektüre des ersten Mondgesangs ${ }^{44}$; es läutet zu Mittag: "Sogleich war die Pflichtübung wie weggewischt." 45 Der Leser im

40 Koestler, Der Mensch - Irrläufer der Evolution, S. 154.

41 H. Bergson, Le Rire, Paris 1969, S. 4; Koestler, Der Mensch - Irrläufer der Evolution, S. 137.

42 Koestler, The Act of Creation, S. 52.

43 Vgl. Goethe: "Das Genie mit Großsinn sucht seinem Jahrhundert vorzueilen; das Talent aus Eigensinn möchte es oft zurückhalten." (Maximen und Reflexionen; in: Sämtliche Werke, Bd. 9, S. 628)

Becketts Auseinandersetzung mit der Genieästhetik kann man durch einen Vergleich seines Gedichtes The Vulture mit Goethes Harzreise im Winter nachvollziehen.

44 Dante, Paradiso, 2. Gesang.

45 S. Beckett, More Pricks than Kicks, London [1931] 1974; Mehr Prügel als Flügel, dt. v. Chr. Enzensberger, Frankfurt a.M. 1989. 
Text - die komplementäre Instanz des Erzählers - kollidiert mit dem Leser des Textes aufgrund zweier "unvereinbarer Verhaltenskodizes". 46

Ich erinnere an den Beginn von Comment c'est, der zu den folgenden Überlegungen anregt. Schreiben ist ein schöpferisches Selbstgespräch; letzteres bildet die Form der Texte und den Ursprung ihrer Inhalte. Die "empathischen Reize" oder die Einfühlung des Lesers und die Vermittlung von Gefühlserlebnissen durch den Erzähler werden degradiert und abgewehrt. Aber das Sprechen behält die Pronomina. Das selbstreflektierende Bewußtsein kollidiert mit Stimmen oder Sprache, die nicht es selbst sind: mit den Inhalten und mit den Grenzen seiner selbst; mit dem Vergessen; schließlich mit der Mühe, sich selbst zu verstehen und auszusprechen. Daß das Dunkel oder das Vergessen als "boue" evoziert werden, ist als Abwehr der Einfühlung aufzufassen. Den Humor kann man als schwarzen oder sadistisch motivierten Humor bezeichnen, weil die inhaltliche Entfaltung des Ich und der übrigen Pronomina als Zerstreuung, als Text-Spiel oder Literatur vorgeführt wird.

comment c'était je cite avant Pim avec Pim après Pim comment c'est trois parties je le dis comme je l'entends

voix d'abord dehors quaqua de toutes parts puis en moi quand ça cesse de haleter racontemoi encore finis de me raconter invocation

instants passés vieux songes qui reviennent ou frais comme ceux qui passent ou chose chose toujours et souvenirs je les dis comme je les entends les murmure dans la boue

en moi qui furent dehors quand ça cesse de haleter bribes d'une voix ancienne en moi pas la mienne

ma vie dernier état mal dite mal entendue mal retrouvée mal murmurée dans la boue brefs mouvements du bas du visage pertes partout ${ }^{47}$

46 Der in der Tradition des französischen Materialismus argumentierende Schiller hat eine ähnliche Kollision wissenschaftlich - mit dem Aha-Effekt - erfaßt: "Der Verstand darf kaum ein wenig auf einer Idee gehaftet haben, so versagt ihm die träge Materie; die Saiten des Denkorgans erschlaffen, wenn sie kaum ein wenig angestrengt worden; der Körper verläßt uns, wo wir sein am meisten bedürfen." (Über den Zusammenhang der tierischen Natur des Menschen mit seiner geistigen; in: F. Schiller, Werke, Großherzog-Wilhelm-Ernst-Ausgabe, Leipzig o.J., Bd. IV, S. 39) 
Der Erzähler dieser Texte formuliert ausschließlich den Erlebnisgehalt, der ihm als Beobachter seiner selbst zugänglich ist. Der Erzähler hat also kaum die "modische Liquidation des Subjekts"48 verfolgt. Er hat vielmehr mit der literarischen Schreibweise ein Problem der Theorie des Geistes untersucht, das mit den Worten Thomas Metzingers darin besteht, "daß eine große Anzahl geistiger Vorgänge einen spezifischen Erlebnisgehalt besitzen, der nur derjenigen Person zugänglich ist, deren innere Prozesse sie sind."49 Beckett hat die Tendenz des literarischen Schreibens nicht als Ausdehnung, sondern als Zusammenziehen aufgefaßt. ${ }^{50}$ Hierbei wird die Vorstellung eines "psychologischen Subjekts"51 nicht außer Kraft gesetzt. Folgt man seinem Aufsatz über Proust, so richtet sich die "désintégration du personnage" 52 gegen die Vorstellung der charakterlichen Identität oder gegen die Vorstellung der Persönlichkeit. Den Inhalt dieser Kritik kann man mit den folgenden Sätzen aus Fritz Mauthners Wörterbuch der Philosophie (1910/11) zusammenfassen:

Der Mensch [hat] in jeder Stunde seines Lebens einen anderen Charakterschwerpunkt als in einer frühern oder spätern Stunde. Ganz unveränderlich ist der Charakter eines Menschen erst nach seinem Tode bestimmt. Er wird von dem Beobachter bestimmt.

Die Persönlichkeit ist gar nichts anderes als die Selbsttäuschung des Ichgefühls oder des Bewußtseins, ist gar nichts anderes als das bißchen Gedächtnis.

Kant und Goethe übersehen beide, daß sie von jeder Person, von jedem Menschen also aussagen, was sie selbstbewußt an ihrer eigenen großen Persönlichkeit erlebt und wonach sie das Maß an die Menschheit gelegt haben, was nur auf große Persönlichkeiten paßt: das ineffable Gefühl, sich selbst allein Zweck und Gesetz zu sein, sprechen sie aus mit dem Wortschalle Persönlichkeit. 53

47 S. Beckett, Comment c'est, Paris 1961, S. 9.

48 Metzinger, Subjekt und Selbstmodell, S. 9.

49 Ebd., S. 20.

50 Beckett, Proust, S. 64.

51 Metzinger, Subjekt und Selbstmodell, S. 21 u. ff.

52 O. Bernal, Langage et fiction dans le roman de Beckett, Paris 1969, S. 147.

53 F. Mauthner, Wörterbuch der Philosophie, Nachdruck Zürich 1980; Stichwort: "Persönlichkeit"; Bd. II, S. 247f., 249, 248. 
Das Konzept, nach dem Beckett seine Erzählfiguren und Ich-Erzähler geschaffen hat, erklärt er im sechsten Kapitel des Romans Murphy: "Amor intellectualis quo Murphy se ipsum amat." Der Erzähler möchte den Ausdruck "Murphys Geist" rechtfertigen. ${ }^{54}$ Es geht hierbei nicht um Murphy als 'wirkliche' Romanfigur, sondern darum, wie er diesen seinen Geist fühlte und ihn sich vorstellte. Dies wird mit einer Definition eingeleitet, die durchaus ironisch das Ausschließen und Ausgeschlossensein erfaßt: "L'esprit de Murphy s'imaginait comme une grande sphère creuse, fermée hermétiquement à l'univers extérieur." Alles, was je im äußeren Universum war, ist und sein wird, soll auch in Murphys Geist gegenwärtig sein: Er bleibt trotzdem nicht im "idealistischen Teer" stecken - und räumt dem Denken oder den Ideen keinen Vorrang gegenüber der sinnlich erfahrbaren Beschaffenheit der Wirklichkeit ein ${ }^{55}$ - , weil er zwischen dem, wovon er eine mentale und physische Erfahrung hat, und dem, wovon er nur eine mentale Erfahrung hat, unterscheidet. Jenen Teil der Erfahrung bezeichnet Murphy als das Wirkliche, das Helle, Klare; diesen Teil als den virtuellen, den dunklen Bereich seines Geistes: Den Fußtritt pflegt er physisch und geistig zu erfahren, also ist er wirklich; die Liebkosung bleibt virtuell - eine Selbstverständlichkeit im "univers beckettien"! Daß nun ein Teil seiner geistigen Erfahrung mit seiner physischen übereinstimmt, verleiht dieser nicht etwa einen höheren Wert gegenüber den rein geistigen Erfahrungen. Sein Geist kann nicht nach ethischen Kriterien, mit dem "ethischen Yo-Yo" oder nach einer beliebigen Wertehierarchie aufgeteilt werden. Er sieht keine Notwendigkeit, "daß sein Licht sein Dunkel

54 Die englische Ausgabe des Romans erschien 1938, die französische 1947; die deutsche Übersetzung von Elmar Tophoven erschien 1959 bei Rowohlt, neu aufgelegt als Bd. 15325 der Reihe rororo. Die folgenden Zitate aus Murphy beziehen sich auf S. 81-85 der französischen und S. 88-93 der deutschen Ausgabe.

55 K.J. Grün, in: Metzler Pbilosophie Lexikon. Begriffe und Definitionen; hg. v. P. Prechtl u. F.-P. Burkard, Stuttgart 1996, S. 225. 
verschlänge"; er ist "mal im Licht [...] mal im Halbdunkel und mal im Dunkel". Hierin liegt eine wichtige Begründung der prinzipiell widersprüchlichen Struktur Beckettscher Texte, in denen philosophischer oder psychologischer Tiefsinn als alberne Trivialität und diese als jener formuliert werden. Wir können ahnen, warum uns Winnie aus Happy Days irritiert und rührt.

Murphy nun vermag die Trennung von Körper und Geist und den "désordre clos" seines Geistes unter der Voraussetzung zu nutzen, daß der Ruhestand der Körperwelt die Bewegung seiner geistigen Welt ermöglicht. Die drei Zonen seines Geistes - Licht, Halbdunkel und Dunkel - können wir als drei Möglichkeiten der Gestaltung der Erzählfiguren oder Ich-Erzähler im Werk Becketts begreifen. Die drei Zonen darf man außerdem als eine umgekehrte Divina Commedia verstehen.

In der Zone des Lichts - des Paradiso - gibt es die "Formen mit Parallelen, ein herrlicher Abriß des Hundelebens": "Ici le plaisir était actif." Hier kann Murphy mit satirischer Tendenz Fußtritte austeilen; er verfügt frei und sich selbstbehauptend über die Wirklichkeit: "das ganze physische Fiasko [wird] zu einem tollen Erfolg"! Ich verstehe das als Verfügungsfreiheit, die der Erzähler und jede seiner nominalen oder pronominalen Projektionen über Semantik und Syntax der Texte besitzt. Die Erzähler Becketts treiben es in der Tat toll mit ihren Puppen, mit deren Namen und Situationen. Denken wir an Hamm, Clov, Nagg und Nell als "ein Spiel für einen Hammer und drei Nägel". ${ }^{56}$ Einen erbarmungslosen Spott treiben die Erzähler mit sich selbst und ihrem Sprechen oder Schreiben: "Rien n'est plus réel que rien." ${ }^{57}$ Der Erzähler blickt in die

\footnotetext{
56 So Beckett nach Ernst Schröder; in: Materialien zu Becketts 'Endspiel', S. 112.

57 S. Beckett, Malone meurt, Paris 1951, S. 32.
} 
"Gummizelle" seiner geistigen Welt ${ }^{58}$, die ihm auch als "Pandämonium" erscheinen kann. ${ }^{59}$

In der zweiten Zone, wo keine Parallelen zwischen Außen und Innen wahrzunehmen sind, widmet Murphy sich der Kontemplation: "Ici le plaisir était esthétique." In beiden Bereichen des Halbdunkels und des Lichts fühlt Murphy sich "souverän und frei" - "Il n'y avait pas de concurrence." Zusätzlich zur inhaltlichen Erfindung verfügen Erzähler und Figuren über die metasprachliche oder metapoetische Reflexionsfähigkeit. Molloy leitet seine Erzählung mit den Worten ein: "Voici mon commencement à moi."60 Hamm kommentiert sein Ende: "jouons ça comme ça".61

Die dritte, schwarze Zone ist der Bereich des Nicht-zu-Fixierenden, des Nichtidentischen; ein Bereich, in dem die Formen zerfließen und ineinander übergehen: "sans cesse s'agrégeant et se désagrégeant [...] sans amour ni haine ni aucun principe de changement concevable. Ici il n'était pas libre, mais un atome dans le noir de la liberté absolue."62

Für den Erzähler der späteren Texte bedeutet dies, daß er ein Medium ist, das die Welt in Außen und Innen teilt: ein vibrierendes Blatt, ein Trommelfell zwischen Schädel und Welt - ein Ich auf dem Papier, ein Text-Wesen. ${ }^{63}$ Der Erzähler versucht, die Wertsetzungen oder Fixierungen seiner Aussagen über die mentale Welt, über seine Träume als Aussagen der mentalen Welt, als Aussagen der Träume erscheinen zu lassen. Er versucht, sich selbst als Instanz vergessen zu machen. Das kann nicht gelingen, weil Druckerschwärze, wenn

58 S. Beckett, Murphy, S. 132: "Les C.M. dépassaient de loin tout ce qu'il avait jamais pu imaginer en fait de paradis d'intérieur." Zur zentralen Bedeutung des Motivs der 'Zelle' im gesamten Werk Becketts vgl. man die präzisen Ausführungen von Gesa Schubert, Das Zylindermotiv in Becketts 'Le Dépeupleur', Magisterarbeit (msch.) am Fachbereich Germanistik der Freien Universität Berlin, Sommersemester 1994, S. 17-19.

59 Beckett, Le Dépeupleur/ Der Verwaiser, S. 50f.

60 S. Beckett, Molloy, Paris 1951, S. 8.

61 Beckett, Fin de partie, S. 112.

62 Beckett, Murphy, S. 84.

63 S. Beckett, L'Innommable, Paris 1992, S. 160. 
man sie als Schrift wahrnimmt, den Anspruch erhebt, "schriftliche Mitteilung eines Menschen [...] zu sein." 64 Vielleicht vermag aber der Leser, über den Text gebeugt, diesen und sich selbst zu vergessen und das Gefühl zu haben, "ein Geschoß ohne Herkunft oder Bestimmung zu sein [...] in der Willenlosigkeit, ein Stäubchen in seiner absoluten Freiheit". 65

Der Erzähler kann diesen Zustand nur als Zusammentreffen zweier unvereinbarer Vorstellungen repräsentieren - des Hierseins und des NichtHierseins: "Assis une nuit à sa table la tête sur les mains il se vit se lever et partir." 66

- Erschienen in: Komik und Solipsismus im Werk Samuel Becketts, hg. v. P.B. u. C. Veit, Stuttgart/Weimar (J. B. Metzlersche Verlagsbuchhandlung ISBN 3-476-45174-7) 1996, S. 239-254.

64 Weimar, Enæy/klopädie der Literaturwissenschaft, \232, S. 129.

65 Beckett, Murphy, S. 85, dt. S. 93.

66 Beckett. Soubresauts, S. 7. 